\title{
A Case of Fatal Mitochondrial Cardiomyopathy
}

\author{
Utako YokoYama, ${ }^{1}$ MD, Toshimitsu ShiBATA, ${ }^{2}$ MD, Kiyoshi Yasui, ${ }^{2}$ MD, \\ Mari IwAmoto, ${ }^{2} \mathrm{MD}$, Kiyohiro TAKIGIKU, ${ }^{2} \mathrm{MD}$, and Shunpei YoKotA, ${ }^{2} \mathrm{MD}$
}

\begin{abstract}
SUMMARY
We report a case of mitochondrial cardiomyopathy in a Japanese boy who presented with severe cardiac heart failure and died 6 days after admission. The onset of mitochondrial cardiomyopathy often occurs very early in childhood and has a rapid downward course. (Jpn Heart J 2002; 43: 61-67)
\end{abstract}

Key words: Cardiomyopathy, Mitochondrial abnormality, Cardiac hypertrophy, Cardiac dilatation, Electrocardiogram, Clinical course

THERE are several diseases known to be mitochondrial abnormalities, namely, mitochondrial myopathy, encephalopathy, lactic acidosis and strokelike episodes (MELAS), myoclonus epilepsy with ragged-red fibers (MERRF), and KearnsSayre syndrome. ${ }^{1-3)}$ Many cases of mitochondrial cardiomyopathies are associated with both myopathy and neuropathy. ${ }^{4}$ Cardiac involvement rarely causes the initial symptoms of mitochondrial cardiomyopathy, and there are only a few reports of such cases. ${ }^{10-13)}$ The onset of mitochondrial cardiomyopathy without extracardiac symptoms occurs very early in childhood, and it often has a rapid downward course. ${ }^{11-13)}$ Frequently, these patients die before a diagnosis can be made.

A 3-year-old Japanese boy weighing $12.6 \mathrm{~kg}(<-1 \mathrm{SD})$ and $95 \mathrm{~cm}$ tall (normal) presented with severe respiratory distress and cyanosis on the first visit to our hospital. He had suffered mild respiratory distress during the previous 5 months without muscle hypotonia or neurological abnormalities. He had an uneventful neonatal period and his family history was unremarkable. He had no developmental delay. His blood pressure was 55/40 $\mathrm{mmHg}$, pulse rate 140 beats/ $\mathrm{min}$, and respiration $65 \mathrm{breaths} / \mathrm{min}$. Auscultation of the heart revealed a gallop rhythm and a systolic regurgitant murmur at the apical region. Mild hepatomegaly was noted. Laboratory studies showed elevated levels of pyruvic acid (1.48 $\mathrm{mg} / \mathrm{dL})$, and creatinine kinase $(172 \mathrm{mU} / \mathrm{mL})$ in plasma. Lactic acid was within

From ${ }^{1}$ the Department of Pediatrics, Saiseikai Yokohama Nanbu Hospital, ${ }^{2}$ the Department of Pediatrics, Yokohama City University School of Medicine, Kanagawa, Japan.

Address for correspondence: Utako Yokoyama, MD, Department of Pediatrics, Saiseikai Yokohamashi Nanbu Hospital, 3-

2-10 Konandai, Konan Ward, Yokohama, Kanagawa 234-8503, Japan.

Received for publication January 5, 2001.

Revised and accepted August 30, 2001. 
normal limits $(12.2 \mathrm{mg} / \mathrm{dL})$. Blood gas analysis revealed marked acidosis with a $\mathrm{pH}$ of 7.168, and base excess values of $-19.1 \mathrm{mEq} / \mathrm{L}$. An antibody test for coxsackie virus was negative and an inflammatory response was not detected. On the chest radiograph, marked cardiomegaly with a CTR of $74 \%$ and pulmonary congestion were observed. The ECG showed regular sinus rhythm with a heart rate of $170 \mathrm{bpm}$ and a normal QRS axis. The PR interval was 0.18 seconds, and the QT interval was 0.41 seconds. Markedly high voltages were noted in the precordial leads, especially in $\mathrm{V}_{5-6}$. Depression of ST segment and T-wave inversion were also observed (Figure 1). Echocardiographic examination revealed marked dilation of the left ventricle (LVIDd: $61 \mathrm{~mm}$ ), and hypertrophy of the left ventricular posterior wall with a thickness of $13 \mathrm{~mm}$ at the mitral valve level. The fractional shortening of the left ventricle was 0.04 . No increase in the brightness of

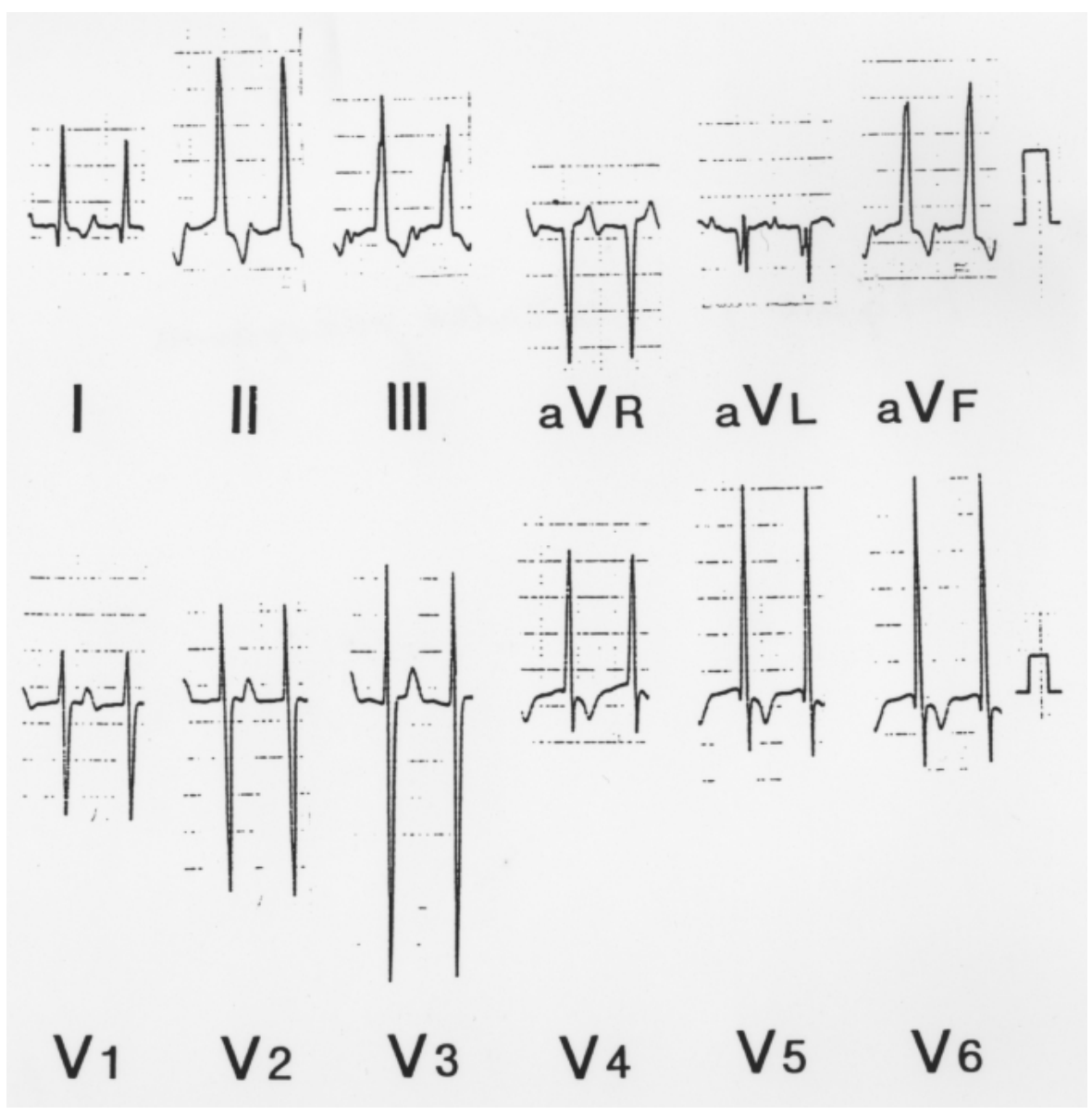

Figure 1. Electrocardiogram showing left ventricular hypertrophy with ST-T wave changes and high voltage in the precordial leads. 
the endocardium was observed. Moderate mitral regurgitation was also observed (Figure 2). Immediately after admission to the ICU, peritoneal dialysis was started because of severe CHF and RF, (BUN $44 \mathrm{mg} / \mathrm{dL}$, serum creatinine 1.66 $\mathrm{mg} / \mathrm{dL}$, GOT $699 \mathrm{U} / \mathrm{L}, \mathrm{LDH} 3360 \mathrm{U} / \mathrm{L})$. Despite partial extracorporeal circulation for 4 days, the cardiac function progressively declined and the patient died 6 days after admission. At autopsy, the heart weight was $270 \mathrm{~g}$ (normal: $78.8 \pm 10.6 \mathrm{~g}$ ) and the pericardial fluid volume was $120 \mathrm{~mL}$. Marked hypertrophy and enlargement of the left ventricle were observed. The thickness of the interventricular septum and left ventricular posterior wall was $11 \mathrm{~mm}$, and that of the right ventriclar wall was $4 \mathrm{~mm}$. Myocardial interstitial fibrosis was not seen in either ventricle. HE staining revealed numerous pink-stained granules, which appeared to be

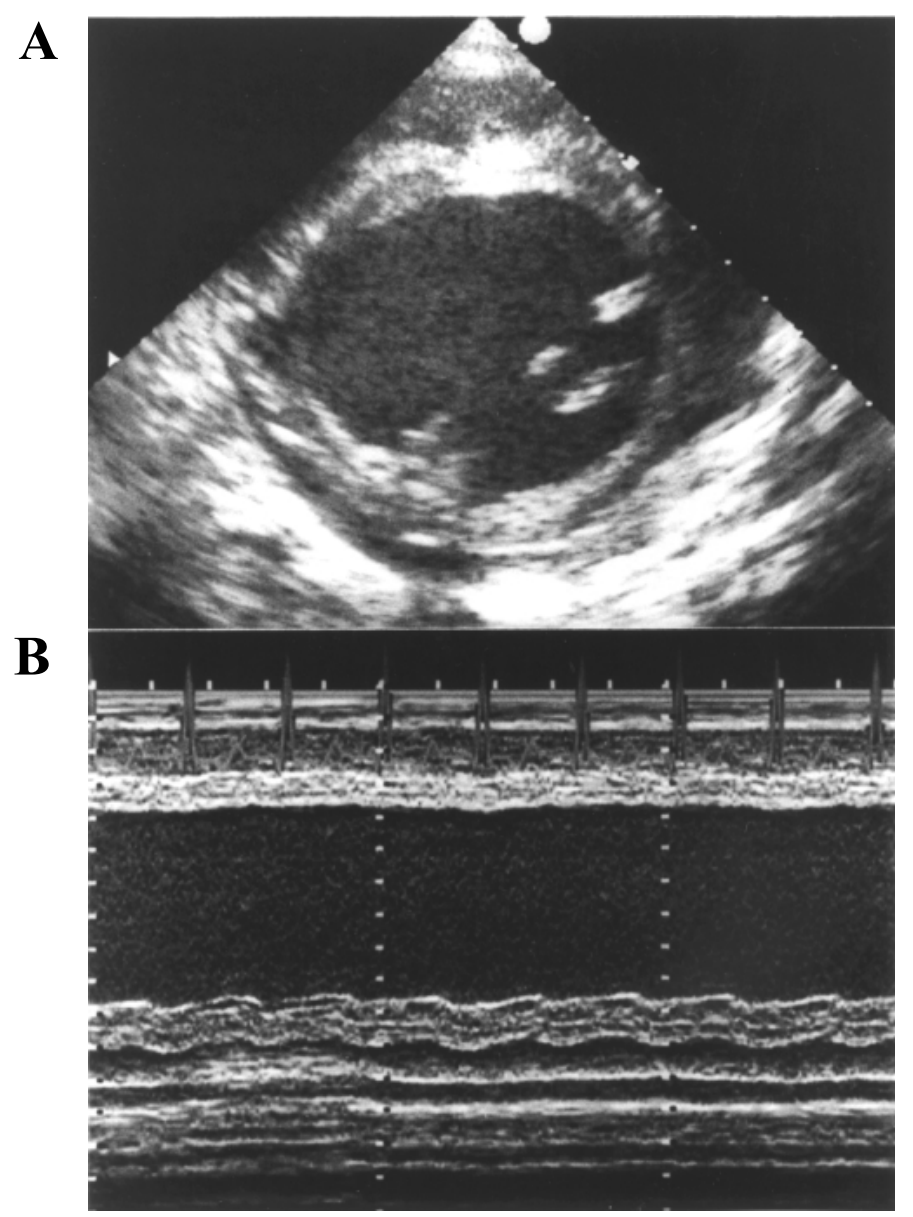

Figure 2. Two-dimensional echocardiogram in the short axis view (A) demonstrates markedly thickened left ventricular walls (LVPW $12.2 \mathrm{~mm}$, IVS $8.8 \mathrm{~mm}$ ), left ventricular dilatation (61 mm) and a moderate amount of posterior pericardial effusion. (B) M mode scan shows that fractional shortening of left ventricle was 0.04 . LVPW=left ventricular posterior wall; IVS=interventricular septum. 
mitochondria, in the myocardial cells (Figure 3). There was no accumulation of glycogen and no anomalies in diaphragmatic, hepatic, or renal specimens. Electron microscopic examination of left-ventricular myocytes revealed many large mitochondria and a marked reduction in myofibril content (Figure 4). Within the

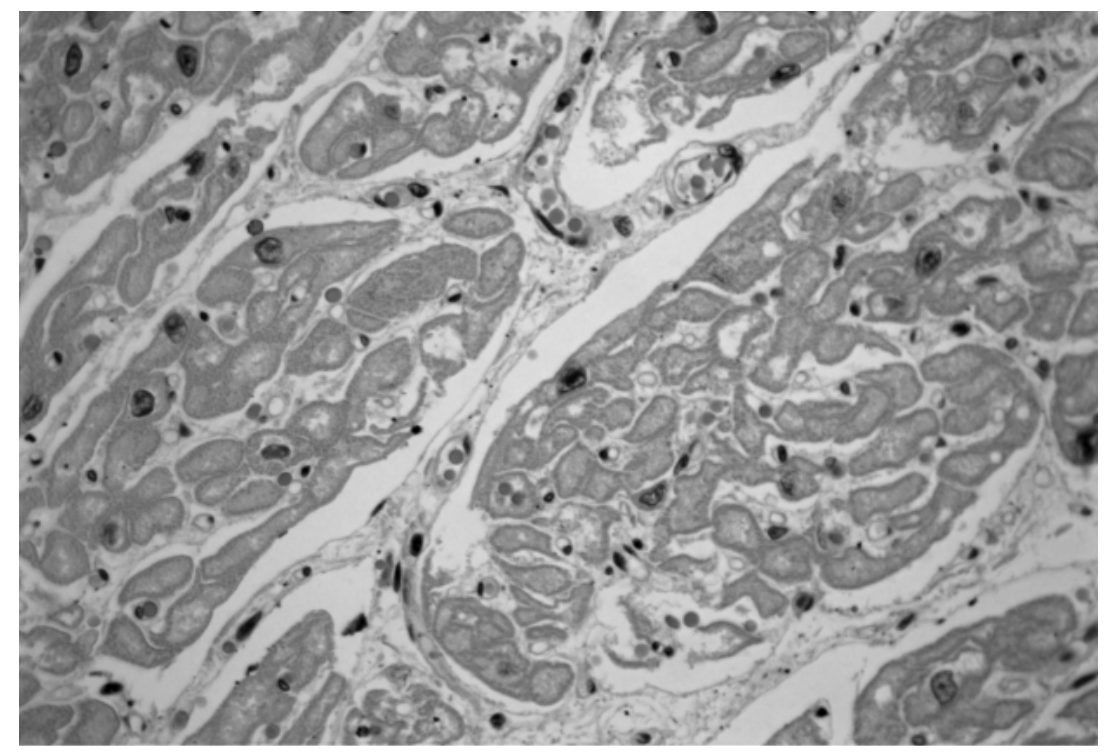

Figure 3. HE staining revealed numerous pink-stained granules, which appeared to be mitochondria, in the myocardial cells.

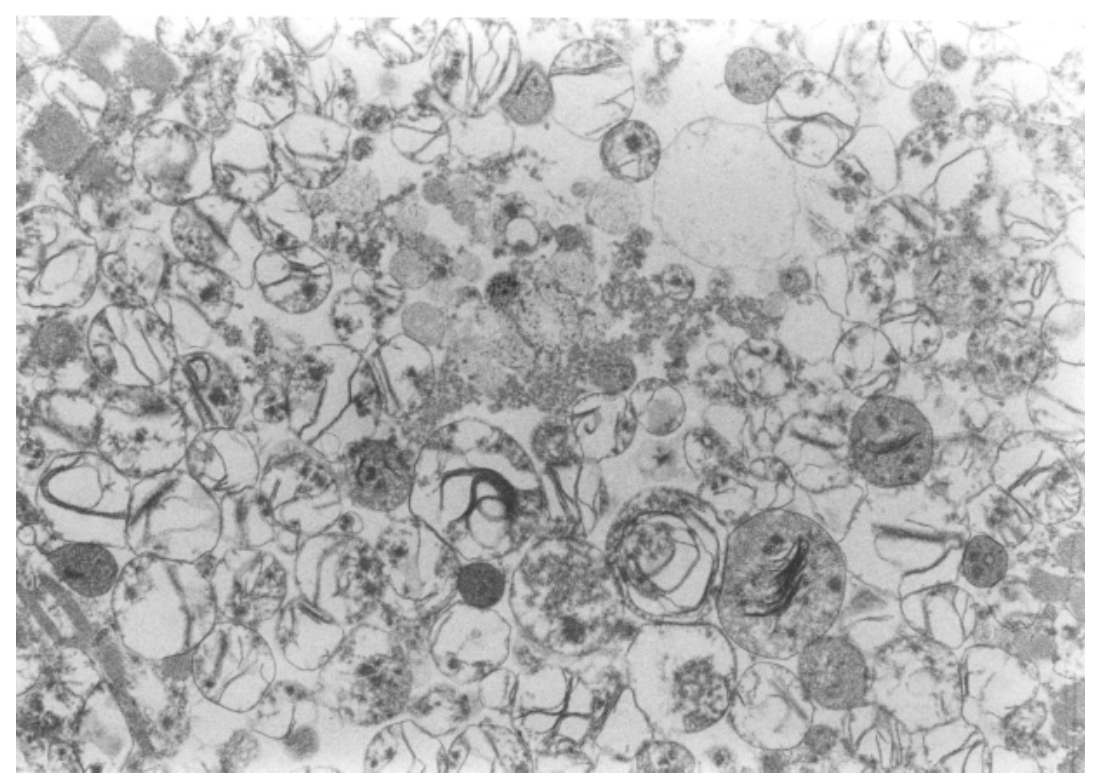

Figure 4. Electron microscopic examination of left-ventricular myocytes revealed many large mitochondria and a marked reduction in myofibril content. 
mitochondria, abnormal multiplication of cristae was also observed. From samples of autopsied ventricular myocardium which were taken within $30 \mathrm{~min}$ after death, the activity of rotenone-sensitive NADH cytochrome-c reductase was 0.15 $\mathrm{mmol} / \mathrm{min} / \mathrm{gm}$ tissue (normal range: $>0.52 \mathrm{mmol} / \mathrm{min} / \mathrm{gm}$ ), indicating complex I deficiency. Cytochrome-c oxidase was $1.23 \mathrm{mmol} / \mathrm{min} / \mathrm{gm}$ (normal range: $>1.41$ $\mathrm{mmol} / \mathrm{min} / \mathrm{gm}$ ) and citrate synthase was $58.07 \mathrm{mmol} / \mathrm{min} / \mathrm{gm}$ (normal range: 7.33-12.43 $\mathrm{mmol} / \mathrm{min} / \mathrm{gm})$. Succinate-cytochrome-c reductase, NADH dehydrogenase, and succinate dehydrogenase were normal. Mutations of mtDNA in left ventricular muscles were also analysed. Previously reported mtDNA mutations at nt3243, 3260, 3303, 4269, 4317, 8344, 9997, and 159517-12) were not recognized by either PCR diagnostic procedures or sequence analysis.

\section{DISCUSSION}

Mitochondrial myopathies, such as MELAS, MERRF, and Kearns-Sayre syndrome, are manifested due to a reduced production of ATP in mitochondria. These diseases sometimes show symptoms associated with conduction abnormality, hypertrophy, or dilated cardiomyopathy. In patients with mitochondrial abnormalities, however, the cardiac abnormalities are often preceded by neurologic and skeletal muscle symptoms of the disease. ${ }^{5)}$ Cases of mitochondrial myopathy which primarily show cardiac symptoms without extracardiac symptoms are extremely rare, except homoplasmic mtDNA mutation which was recently reported by Shin, et al where all corresponding patients have cardiac manifestation only. ${ }^{10,11,13,14)}$ Furthermore, in approximately half the number of cases of mitochondrial myopathy where left ventricular hypertrophy is present, including cases of MERAS and MERRF, death occurs from heart failure usually within the first year of life. $\left.{ }^{6}\right)$ Some cases of cardiac dysfunction due to mitochondrial abnormalities have very early onsets and rapid downward courses, while other types of cardiomyopathy with homoplasmic mtDNA mutation show gradual onset and process. ${ }^{2,14}$ When mitochondrial diseases are not associated with neurologic or skeletal muscle symptoms, it is extremely difficult to distinguish a cardiomyopathy due to mitochondrial abnormalities from other types of cardiomyopathies. Mitochondrial cardiomyopathies that can be confused with other types of cardiomyopathy include dilated cardiomyopathy, primary endocardial fibroelastosis, anomalous origin of the left coronary artery from the pulmonary artery, and the metabolic disorder Pompe's disease. Mitochondrial cardiomyopathies are not uncommonly diagnosed and sometimes treated as idiopathic hypertrophic cardiomyopathy and/or dilated cardiomyopathy. When unexplained cardiomegaly and/or myocardial hypertrophy are detected, in particular in the presence of short stature, hypotonia, and cataracts, mitochondrial cardiomyopa- 
thy should be considered as a differential diagnosis. Furthermore, the measurement of blood lactic acid and pyruvic acid levels and blood gas analysis should be mandatory in order to determine whether the patient has lactic acidosis.

Mitochondrial cardiomyopathy is commonly associated with an elevated creatinine kinase level, marked hypokinesis of the left ventricle on a 2D echocardiogram, and findings of high voltages and ST depression. In the present case, marked by high voltages and $\mathrm{T}$ wave inversion, these findings were also observed. In addition, the prolongation of the PR interval $(0.18 \mathrm{sec})$ suggested atrioventricular conduction delay. Such findings in the present case appeared to be typical, although ECG findings of mitochondrial cardiomyopathy with primary cardiac symptoms have been rarely reported. ${ }^{13)}$

NADH-ubiquinone reductase (complex I), succinic acid-ubiquinone reductase (complex II), ubiquinone-cytochrome-c reductase (complex III), cytochrome-c reductase (complex IV), and ATP synthase (complex V) are found in the inner mitochondrial membrane. In many cases, there are abnormalities in complex I and complex IV. The biochemical tests indicated decreased activity of respiratory chain enzymes. Genes encoding proteins found within mitochondria are located in the cell nucleus as well as within the mitochondrial cytoplasm. The A-to-G 3243 tRNA ${ }^{\text {Leu (UUR) }}$ point mutation is observed in $83 \%$ of MELAS cases. Seventy-nine percent of MERRF cases are found to contain an A-to-G 8344 tRNA ${ }^{\text {lys }}$ point mutation. These point mutations in mitochondrial DNA have also been linked to other types of cardiomyopathy. ${ }^{6}$ In addition, several reports indicate that other genetic abnormalities in mtDNA cause cardiopulmonary arrest and hypertrophic cardiomyopathy. These abnormalities include an A-to-G 3260 tRNA $^{\text {leu (UUR) }}$, a C-to-T 3303 tRNA ${ }^{\text {leu(UUR) }}$, an A-to-G 4317 tRNA $^{\text {Ile }}$, an A-to-G 4269 tRNA $^{\text {Ile }}$, an A-to-G 15951 tRNA $^{\text {Thr }}$, and a T-to-C 9997 tRNA $^{\text {glycine }}$. $^{712)}$

The patient died before a diagnosis could be made because of poor extracardiac symptoms. When unexplained cardiomegaly or myocardial hypertrophy are detected, we should consider mitochondrial cardiomyopathy as a differential diagnosis. Though mtDNA abnormalities were not detected, we considered the case to be mitochondrial cardiomyopathy based on the elevated levels of pyruvic acid and complex I deficiency. The present case and several reports indicate a number of unique features of fatal mitochondrial cardiomyopathy as follows. The onset of mitochondrial cardiomyopathy without extracardiac symptoms occurs very early in childhood, and often has a rapid downward course, ${ }^{11-13)}$ cardiac dilatation and hypertrophy are simultaneously observed with hypokinesis, ${ }^{7)}$ markedly high voltages and ST depression are seen in the ECG ${ }^{13)}$ abnormal multiplication of mitochondria is observed in the myocardial tissue, ${ }^{5,13}$ blood lactic acid and pyruvic acid levels are elevated, ${ }^{5)}$ and the activity of respiratory chain enzymes is reduced. ${ }^{3,48}$ Several mtDNA abnormalities have already been reported. ${ }^{5,7-12)}$ 


\section{ACKNOWLEDGMENTS}

This study was supported by Rumiko Matsuoka and Michiko Furutani of Tokyo Women's University of Medicine.

\section{REFERENCES}

1. Wallace DC. Mitochondria disease in man and mouse. Science 1999; 283: 1482-8.

2. Wallace DC. Mitochondrial defects in cardiomyopathy and neuromuscular disease. Am J Heart 2000; 139: S70-S85.

3. Goto Y, Nonaka I, Horai S. A mutation in the t-RNALEU (UUR) gene associated with the MELAS subgroup of mitochondrial encephalomyopathies. Nature 1990; 348: 651-3.

4. Ito T. Molecular biology of mitochondrial DNA and mutation in mitochondrial cytopathy. [in Japanese] Nippon Rinsho 1993; 51: 1425-8.

5. Marcy LS, Gerald FC, Angela EL, et al. Clinical approach to genetic cardiomyopathy in children. Circulation 1996; 94: 2021-38.

6. Anan R, Nakagawa M, Miyata M, et al. Cardiac involvement in mitochondrial diseases: a study on 17 patients with documented mitochondrial DNA defects. Circulation 1995; 91: 955-61.

7. Tanaka M, Obayashi T, Yoneda M, Kovalenko SA, Sugiyama S, Ozawa T. Mitochondrial DNA mutation in cardiomyopathy: combination of replacements yielding cysteine residues and tRNA mutations. Muscle and Nerve Suppl 3. 1995; S165-S169.

8. Massimo Z, Caterina M, Carlo A, Giovanni MF, Pierre R, Alessandro P. Oxphos defects and mitochondrial DNA mutations in cardiomyopathy. Muscle and Nerve Suppl 3. 1995; S170-S174.

9. Taniike M, Fukushima H, Yanagihara I, et al. Mitochondrial tRNA ${ }^{\text {Ile }}$ mutation in fatal cardiomyopathy. Biochem Biophys Res Commun 1992; 186: 47-53.

10. Ozawa T, Katsumata K, Hayakawa M, Tanaka M, et al. Genotype and phenotype of severe mitochondrial cardiomyopathy: a recipient of heart transplantation and the genetic control. Biochem Biophys Res Commun 1995; 207: 613-20.

11. Frank M, Ingrid T, Lee B, Brian HR. Maternally inherited hypertrophic cardiomyopathy due to a novel T- to- C transition at nucleotide 9997 in the mitochondrial tRNA ${ }^{\text {glycine }}$ gene. Am J Hum Genet 1994; 55: 437-46.

12. Silvestri G, Santorelli FM, Shanske S, et al. A new mtDNA mutation in the tRNA ${ }^{\text {Leu (UUR) }}$ gene associated with maternally inherited cardiomyopathy. Human Mutation 1994; 3: 37-43.

13. Alexander P, Harry BN, Salvatore D, Robert S, Nereo B. Histiocytoid cardiomyopathy of infancy: deficiency of reducible cytochrome b in heart mitochondria. Pediatric Research 1984; 18: 1023-8.

14. Shin WS, Tanaka M, Suzuki J-I, Hemmi C, Toyooka T. A novel homoplasmic mutation in mtDNA with a single evolutionary origin as a risk factor for cardiomyopathy. Am J Hum Genet 2000; 67: 1617-20. 